\title{
El papel de la familia de la persona adulta con discapacidad en los procesos de inclusión laboral: Un reto para la educación especial en Costa Rica
}

\author{
The Role of the Adult with Disabilities' Family in the Labor Inclusion Processes: A \\ Challenge for Special Education in Costa Rica
}

\author{
Ana Magaly Madrigal-Lizano' \\ Universidad Nacional \\ División de Educación Básica \\ Heredia, Costa Rica \\ magaly.madrigallizano@gmail.com
}

Recibido 13 de junio de 2014 • Corregido 16 de febrero de 2015 • Aceptado 27 de abril de 2015

\begin{abstract}
Resumen. El siguiente ensayo presenta las reflexiones de la autora, acerca de los impactos familiares positivos y negativos en los procesos de inclusión laboral, de la persona adulta con discapacidad, la significancia y desafíos que debe asumir el personal docente a cargo de los servicios de educación especial y los retos de los entes de educación superior en la formación de formadores, sobre la responsabilidad de establecer la mayor cantidad de mecanismos posibles, para asumir conjuntamente con el grupo familiar los procesos, espacios, formación y la posible toma de decisiones de la persona con discapacidad en relación con su futuro laboral y las habilidades necesarias y requeridas para que asuma un estilo y calidad de vida lo más autónomo e independiente. El ensayo se enmarca en el modelo social dentro de un paradigma inclusionista enmarcado en un enfoque de derechos humanos. En concordancia con Vargas (2012) plantea el modelo social, donde destacan las barreras culturales y el entorno, con una visión holística, que aunque no niega la intervención individual enfatiza en la inclusión en un mundo que ha sido diseñado por personas "sin discapacidad" y para estas. Al pasarse del modelo médico al social, se traslada la responsabilidad a los ámbitos sociales y políticos y, desde esa perspectiva, es la familia, entonces, un pilar indiscutiblemente relevante para ser considerado en los diferentes ámbitos de formación, capacitación e información en pro de la persona adulta con discapacidad, que requiere y merece la oportunidad del trabajo como paso y puente en el logro de una vida autónoma e independiente.
\end{abstract}

Palabras claves. Persona con discapacidad, equidad, derechos humanos, trabajo, familia, barreras, inclusión.

\footnotetext{
${ }^{1}$ Es actualmente académica de la Universidad Nacional de Costa Rica. Ofrece consultorías en temáticas relacionadas con la inclusión laboral de personas adultas con discapacidad a organizaciones como Programa de Naciones Unidas PNUD, Agencia de Cooperación Técnica Alemana GIZ. Trabaja en equipo para el Consejo de la Persona Joven CPJ, en cursos relacionados a habilidades blandas para adultos con discapacidad. Ha trabajado para el Ministerio de Educación Pública por 12 años en servicios de apoyo en educación especial y ha producido documentos y manuales de apoyo vinculados a la capacitación y estrategias de trabajo, en procesos de inclusión laboral de adultos con discapacidad, así como guías de apoyo para docentes y posibles empleadores. Su campo de especialización es la educación especial con énfasis en integración y actualmente cursa una maestría en diversidad en los procesos pedagógicos. Ha participado en seminarios, coloquios, talleres y otras actividades tanto a nivel nacional e internacional. Colabora, como académica participante en proyectos de investigación y extensión de la Universidad Nacional. En dichos procesos de inserción laboral se articulan las bolsas de empleo, los gobiernos locales y las iniciativas tanto gubernamentales y del sector privado, en materia de adultos y discapacidad en relación con el empleo.
} 


\begin{abstract}
The following essay presents the author's thoughts regarding positive and negative family impacts of the process of including the adult with disabilities. It also discusses the significance and challenges faced by those teachers and schools in charge of special education based on the responsibility of establishing the most mechanisms possible to provide, along with their families, a free environment when it comes to decision making for the population with disabilities in regards to their job future and the special skills needed to provide a free independent lifestyle. The essay focuses on a social model in an inclusive paradigm based on a human rights perspective. In accordance with Vargas (2012), it suggests a social model that highlights social barriers and their environment with a holistic view, which does not exclude individual intervention but emphasizes the inclusion of this population in a world which has been designed by and for people that do not experience any disability. When shifting from the medical model to the social one, responsibility is also transferred to the social and political aspects, hence making the family a relevant pillar to be considered in different training and information environments in support of the adult with disabilities who needs and deserves the opportunity of having a job as a way to be autonomous and independent.
\end{abstract}

Keywords. Person with disabilities, equity, human rights, labor, family, barriers, inclusion.

\title{
Para iniciar
}

Actualmente en Costa Rica la inclusión laboral busca la contratación de personas con discapacidad en diferentes puestos y empleos que provean salarios dignos, con todas las garantías de ley, según lo que el Código de Trabajo establece. Tales acciones representan uno de los retos más significativos del país. A través de una iniciativa por parte del Ministerio de Trabajo y Seguridad Social (MTSS), en conjunto con la Oficina de Coordinación de Naciones Unidas, el PNUD y la OIT (Organización Internacional para el Trabajo) se crea El plan nacional de inserción laboral para la población con discapacidad en Costa Rica, como resultado de la aplicación de la metodología denominada "Marco de aceleración de los objetivos del milenio" del 2012 al 2015 (Ministerio de Trabajo y Seguridad Social de Costa Rica, 2012), para apresurar el cumplimiento de la meta 1.3

Lograr empleo pleno productivo y trabajo decente para todos, incluyendo mujeres y jóvenes", enfocando el esfuerzo en la aceleración del acceso al empleo a la población con discapacidad, para la cual las dificultades de encontrar empleo productivo y decente es mayor que el promedio nacional. (p. 17)

Según el censo realizado en Costa Rica por el Instituto Nacional en Estadística y Censos (INEC) (2011), se cuenta con total de 24408 personas con discapacidad que no tienen trabajo. Una lucha por establecer mayores condiciones de equidad, para esta población, representa un cambio actitudinal y de formación, para una nueva generación de docentes y de padres y madres de familia, que manifiesten compromiso con la autonomía e independencia de sus estudiantes e 
doi: http://dx.doi.org/10.15359/ree.19-2.12

URL: http://www.una.ac.cr/educare

hijos e hijas. La familia, indiscutiblemente, viene a determinar, en forma significativa, los niveles de éxito con los que puede contar ese hijo e hija en su futuro y su posible inserción en el ámbito laboral. En Costa Rica, gran parte de los núcleos familiares que cuenta con un miembro en condición de discapacidad mantiene prácticas donde predominan mitos y estigmas negativos y desfavorables en la forma en que le visualizan. Ello ha implicado que sienta la libertad de asumir las decisiones más importantes de sus hijos e hijas, ya sea por motivos justificados o injustificados.

Gutiérrez (2006) plantea la realidad de la sobreprotección en la familia de las personas con discapacidad, como un evento donde "con la mejor de las intenciones suelen sobreproteger a estos hijos e hijas y con ello disminuyen sus posibilidades de relación. Dicha sobreprotección suele provocar un empobrecimiento del entorno, además de ejercer control mucho mayor de las conductas, especialmente las que consideran peligrosas... (p. 18).

Esto genera una limitante importante, pues gran cantidad de esta población, con las competencias necesarias para adquirir puestos de trabajo, no los logra por impedimentos de carácter familiar. Abocarse al análisis de esta situación es pertinente y, por ello, el presente ensayo expone las principales barreras que experimenta el adulto y la adulta con discapacidad, en los procesos de inclusión laboral y la significancia que representa la familia con respecto a los apoyos que esta puede brindar.

\section{Padadigmas, familia y discapacidad}

Según Córdoba, Mora, Bedoya y Verdugo (2007, p. 30) se define la familia como"ecosistema, por cuanto ésta permite percibir a la familia dentro de un amplio espectro, el cual abarca a todos los miembros de la familia y a los agentes extemos que como vecinos, amigos, comunidad, pueden tener algún efecto en la interacción familiar".

Podemos decir, entonces, que a partir de la concepción de familia como un ecosistema, en el que los miembros que la conforman se influyen entre sí, la persona adulta con discapacidad los impacta directamente, desde su condición de discapacidad, con todo lo que esta representa por sí misma, como son los cuidados a nivel de salud, los tratamientos diarios, las rutinas educativas desde edades tempranas, los esfuerzos en la participación de la persona con discapacidad en los diferentes entornos sociales y, por consiguiente, su inclusión plena en este entorno y todo lo demás que se desarrolla en el diario convivir de cualquier dinámica familiar. La familia, a su vez, proyecta en la persona acciones específicas tanto positivas como negativas, según la manera en que la asume y la visualiza.

A lo largo de todos los procesos de inclusión laboral en los que he participado por medio del Proyecto UNA Oportunidad de empleo (2011-2013) , las prácticas negativas más sobresalientes a nivel familiar son la sobreprotección, el control estricto en los horarios sobre lo que debe o no 
doi: http://dx.doi.org/10.15359/ree.19-2.12

URL: http://www.una.ac.cr/educare

CORREO: educare@una.cr

debe hacer la persona con discapacidad durante el día, las actividades de recreación en caso de existir son valoradas o escogidas por los encargados o encargadas y, en ciertos casos, se puede ir a los extremos del abandono total, lo cual se destaca como violencia intrafamiliar, pues constituye la negación de los derechos para que ese ser se desarrolle plenamente.

Gutiérrez (2006) define la violencia como todo lo que se hace o se deja de hacer, ya sea por un motivo que responde a la ignorancia, descuido o incapacidad, tanto en un ámbito familiar como social. En el caso de las personas con discapacidad la violencia intrafamiliar puede responder a cosas que no se hacen o se dejan pasar y que niegan al adulto sus derechos y libertades impidiendo su desarrollo pleno.

Así también, positivamente, se observa a la familia ejerciendo un papel de ente motivador ante los desafíos laborales que se le presentan a la persona adulta con discapacidad, confiando en él o ella a pesar de existir diversas deficiencias, posibilitando de esta manera que pueda tomar control sobre su propia vida. Escoger un deporte, trabajar, asumir estudios y hasta formar un hogar son algunos buenos ejemplos de lo que puede lograr el sujeto al contar con el debido apoyo familiar.

Como parte de mi práctica profesional en el campo de la educación especial y de los procesos de inclusión laboral que he llevado a cabo en empresas públicas y privadas, he observado, en las familias, los esfuerzos de ubicación laboral de jóvenes con discapacidad. Es importante mencionar, en este momento, y como se aclaró en la introducción, que se apunta hacia un modelo social, el cual responde a un paradigma inclusionista, enmarcado en un enfoque de derechos humanos.

Según López, (2011), este es caracterizado principalmente por la promoción de la independencia y la autonomía por parte de las personas con discapacidad, en asumir la dirección de su propia vida y ser partícipes de una sociedad accesible.

Dentro de los objetivos principales de este modelo social están: validar la discapacidad como una construcción social, donde todos somos responsables de brindar las condiciones equitativas para esta población; reconocer que la discapacidad es una condición del entorno y la persona que la vive no es responsable o culpable de presentar una deficiencia; son por lo tanto los espacios y entornos quienes imponen las barreras, de allí que la persona pueda verse más incluido o más violentado en su acceso a la sociedad y en su participación en esta.

Finalmente el modelo social visualiza a la persona con discapacidad como un sujeto de derechos, capaz y con autoridad de manejar y decidir en su vida, con absolutos o elevados niveles de autonomía e independencia, lo cual conlleva a un objetivo final relevante: asumir responsablemente el control de su vida.

Lejos de las descripciones anteriores, la realidad en Costa Rica muestra a gran cantidad de familias, dirigiéndose a su familiar con discapacidad desde modelos tradicionales y rehabilitadores, los cuales se especifican a continuación. 
Para López (2011), el modelo tradicional está caracterizado por la segregación, la dependencia y la concepción de la persona con discapacidad como objeto de asistencia y protección. El asistencialismo es la característica más sobresaliente en este modelo y generalmente la realidad nos muestra a madres, más que padres u otros miembros, decidiendo que su hijo e hija no es capaz de trabajar, en tanto él o ella no le pueden acompañar al puesto de trabajo. Desde mi actual experiencia en procesos de inclusión laboral, aplico múltiples entrevistas a personas jóvenes adultas con discapacidad para la adquicisión de un puesto de trabajo. Generalmente llegan en compañía de sus madres y hermanas, y se percibe que son estas últimas, generalmente, las que al final del proceso toman las decisiones por esta población, sobre su realidad laboral. En estas situaciones no se pretende dejar fuera a la familia; sin embargo, lo que deseamos en todo momento es que la persona con discapacidad se encamine a una vida independiente y autónoma, donde sea reponsable sobre las decisiones, ya sean estas positivas o negativas, como en el caso de cualquier ser humano, con o discapacidad sin esta, que tiene derecho a un empleo digno.

Aclaro que este ensayo se enfoca en aquellos adultos que cuentan con las habilidades funcionales y competencias necesarias para adquirir un puesto de trabajo. Dichas competencias se enmarcan en el manejo y asertiva aplicación de las habilidades blandas. Según Silva (2013), las habilidades blandas son:

Aquellas orientadas al desarrollo de aptitudes sociales. Son reconocidas de mejor manera por los empleadores como un estudiante, joven o trabajador busca empleo. Se trata de capacidades comunicativas, de trabajo en equipo, flexibilidad y adaptabilidad frente a un determinado trabajo. (p. 22)

No se omite, por lo tanto, la realidad de gran cantidad de jóvenes que no pueden acceder a un puesto de trabajo, por motivo de un elevado compromiso a nivel cognitivo, sensorial, físico o mental.

Por su parte López (2011), con respecto al modelo de rehabilitación, afirma que este sobresale cuando la familia sigue visualizando la discapacidad no como una condición, sino como una enfermedad, la cual es controlada y determinada en todas las áreas de la vida, por las recomendaciones que da el personal médico o especialistas. La discapacidad se asume como un problema de carácter individual y no una responsabilidad y construcción social, por lo que la persona con discapacidad debe obtener asistencia por parte de otras, en cada cosa en que evidencia una necesidad, cuando lo idóneo es que ella misma adquiera las habilidades para autogestionar la solución a las diferentes barreras u obstáculos que le presenta el entorno y la vida como tal. 
doi: http://dx.doi.org/10.15359/ree.19-2.12

URL: http://www.una.ac.cr/educare

CORREO: educare@una.cr

\section{Afrontar el presente y planear el futuro}

Desde las perspectivas especificadas anteriormente, las familias tienen dos grandes desafíos: afrontar el presente y planear el futuro.

Afrontar el presente conlleva reconocer que su familiar con discapacidad, después de finalizar períodos de estudio en instituciones de educación especial o servicios en modalidades vocacional u ocupacional, está destinado a pasar la mayor cantidad de tiempo realizando tareas domésticas en casa, dando como resultado final una proporción de tiempo libre muy elevada, la cual se convierte en un factor de riesgo muy alto y existe el riesgo de empezar a mostrar diversos síntomas de ansiedad y comprometedores estados de ánimo. Los encargados afirman vivenciar una gran preocupación y estrés al no saber cómo enfrentar la posibilidad de conseguir un empleo para que su hijo o hija trabaje, lo cual los conduce a tomar muchas veces la decisión de no permitir o dar oportunidad a un posible proceso de adquisición de empleo.

Esta situación de enfrentar el presente también está alimentada de una serie de mitos y estereotipos que giran negativamente alrededor de la persona con discapacidad.

Partimos del hecho de que la sociedad y, principalmente la familia, les ve como niñas y niños eternos, castrándoles la posibilidad de tener las herramientas necesarias para salir a la calle y movilizarse con autonomía eindependencia. Pormuchotiempo seles ha valorado con incapacidad de sentir, aprender y desarrollar proyectos personales con éxito. El trabajo es uno de estos y quienes primero obstaculizan su desarrollo en el campo laboral, y en decidir unilateralmente, si tienen opción de inserción laboral, son sus propias familias. En estas, principalmente, las madres asumen la potestad y se adjudican el poder de determinar si la persona adulta con discapacidad está en condición de adquirir un empleo y mantenerlo con éxito.

Es importante aclarar, en este punto, que según las experiencias en el Proyecto UNA Oportunidad de Empleo (2011-2013) (Holst y Madrigal, 2014), los encargados y encargadas al final muestran gran alegría ante la oportunidad del trabajo en sus hijos e hijas, pero previo a la demostración de placer, han demostrado frustración por tantas ocasiones en que les han cerrado las puertas ante posibles solicitudes de empleo, enojo por el rechazo mostrado a nivel social ante las capacidades de sus hijos e hijas, inseguridad por la posibilidad que algo malo les ocurra en el camino, dependencia por el qué voy a hacer de mi vida ahora que no va estar en casa y desconfianza sobre el éxito real en el futuro empleo de su hijo o hija .

En intercambios informales con padres y madres de familia se han recopilado frases acerca de las posibilidades de que su hijos trabajen, las cuales denotan preocupación y entre sus argumentaciones mencionan "que les da miedo que le hagan daño", "que no son capaces de movilizarse independientemente en otros espacios, sin la presencia de ellos", "que son muy ingenuos e inocentes, propensos a que cualquier otro les engañe" y todas las afirmaciones anteriores, 
responden a largos procesos de nula formación y capacitación adecuada por parte de profesionales incapaces de brindar, a las familias, la motivación de ver a sus hijos e hijas como personas con opciones, sueños y alternativas para un desarrollo más integral.

La sobreprotección, como un tipo de violencia familiar emitida por quienes les cuidan como sobreprotección y la persona adulta con discapacidad como sobreprotegida, conlleva a desarrollar e incrementar una serie de conductas, que encaminan, al primer sujeto, a incapacitar a la persona con discapacidad, hacerla cada vez más dependiente, no dejarla que experiemente cosas nuevas y que fracase como cualquier otro ser humano, lo transforma en un ser que desconoce lo que le rodea o lo que le conviene $y$, de esta manera, puede ser muy fácil violentar sus derechos.

Para Gutiérrez (2006, citando a Quirós), la comunicación entre las familias es prioritaria, en tanto es importante que:

[Las personas con discapacidad] sean informadas sobre lo que ocurre a su alrededor, si la persona aún no ha desarrollado un lenguaje verbal, se debe optar por otras alternativas como los pictogramas, objetos concretos o señas que le proporcionarán una oportunidad de comunicación, lo que impactará en su ambiente inmediato modificándolo, expresando así sus sentimientos y preferencias, lo que les permitirá ejercer sus derechos. (p. 60)

La persona con discapacidad como figura sobreprotegida se asume y actúa como una persona dependiente, insegura, temerosa, incapaz de resolver problemas, con dificultades intra e interpersonales y elevados niveles de frustración, lo cual le dificulta entender de dónde provienen.

Parece, entonces, que las herramientas positivas, con las que cuenta la familia para enfrentar el presente de su familiar con discapacidad, son casi nulas o muy poco alentadoras. En la medida que se asuma la familia como una pieza clave en los procesos de educación y de inclusión laboral, esta debe ser informada, capacitada y transformada, para trabajar intensamente en los cambios y realidades que el paradigma social nos propone en la vida del adulto con discapacidad.

El enfrentar positivamente el presente hará más fuerte e independiente a ese joven y más libre y segura a esa familia.

Por otra parte, planear el futuro de la persona con discapacidad es sinónimo de evitar una posible crisis relacionada con su cuidado y calidad de vida. Esto debe hacerse mucho antes de que el cuidador o cuidadora sufra de una crisis por salud, fallezca y no puedan preveer los apoyos necesarios posteriormente. Para este momento, es necesario que el individuo adulto con discapacidad trabaje, se asista de manera independiente, resuelva posibles conflictos que se le presentan y demuestre, por sí mismo, mantener una adecuada calidad de vida. 
doi: http://dx.doi.org/10.15359/ree.19-2.12

URL: http://www.una.ac.cr/educare

CORREO: educare@una.cr

Se podría decir que este es un proceso de larga capacitación de parte de educadores y profesionales a cargo, pero sobre todo de la familia, la cual tiene la última palabra con respecto a las oportunidades laborales que se pueden presentar a su miembro.

Al Estado, la sociedad, pero sobre todo el personal docente les corresponde la responsabilidad de ir generando el cambio en la visión actual y en la realidad de muchas familias que tienen en sus hogares una persona con discapacidad. En la medida en que el joven se independiza, la familia también crece, adquiere procesos de autonomía familiar, ayuda a mejorar las situaciones económicas familiares y permiten el acceso a nuevas oportunidades y posibilidades que engrandecen su dignidad y calidad de vida.

Para Verdugo (2000) la calidad de Vida se define como:

Las condiciones de vida deseadas por una persona en relación con ocho necesidades fundamentales que representan el núcleo de las dimensiones de la vida de cada uno: bienestar emocional, relaciones interpersonales, bienestar material, desarrollo personal, bienestar físico, autodeterminación, inclusión social y derechos. (p. 5)

Como podemos visualizar, todas las necesidades fundamentales condicionadas con la calidad de vida se relacionan directamente con el derecho y el acceso al trabajo, por lo que cualquier barrera que atente contra estos, afecta significativamente la calidad de vida de la persona con discapacidad.

El Artículo 8 de la Ley 8661 Toma de conciencia, sobre los derechos de las personas con discapacidad expresa:

1. Los estados Partes se comprometen a adoptar medidas inmediatas, efectivas y pertinentes para:

a) Sensibilizar a la sociedad, incluso a nivel familiar, para que tomen mayor conciencia respecto de las personas con discapacidad y fomentar el respeto de los derechos y la dignidad de estas personas. (Asamblea Legislativa de la República de Costa Rica, 2008, p. 4)

La Convención de los Derechos de las personas con discapacidad, tambien ratificada mediante la ley 8661 de Asamblea Legislativa de la República de Costa Rica. (2008), en su Artículo 27 en lo medular plantea el derecho al trabajo y empleo de los adultos con discapacidad. Se tiene claro desde todo marco legal y humano que el trabajo es un derecho y como derecho, la posibilidad de ejercerlo. Esto es una linda realidad en el papel, sin embargo, muchas personas adultas con discapacidad siguen sin empleo por falta de oportunidades laborales y por la negación de sus propias familias a que lo hagan. 
Leach (2002) menciona que:

El trabajo es probablemente, el indicador más importante de la independencia de una persona y su contribución a la sociedad, nos guste o no. Conseguir un trabajo, es la base para la autoestima personal y la capacidad para llevar una vida independiente en [la] sociedad (p. 17).

\section{Educación especial y la familia de la PCD}

A partir del concepto anterior, planteo la enorme responsabilidad que, en materia de inclusión laboral, tiene la educación especial en Costa Rica. Siempre en los conversatorios formales e informales con padres y madres de familia, encontramos un divorcio entre el papel que asumen y lo que docentes de educación especial hacen. Los profesores y profesoras de educación especial se disgustan con los elevados niveles de sobreprotección de los padres y madres y este sector, a su vez, percibe e interpreta al cuerpo de docentes de educación especial como profesionales especialistas en diversas áreas, que no les toman en cuenta, a pesar de conocer a la persona con la que trabajan desde que nació.

Los padres y madres afirman tener mucha información que compartir con docentes acerca de sus hijos e hijas; sin embargo, esa solicitud casi nunca llega, solo a inicio de año para contestar, si es el caso, las entrevistas iniciales. Las Normas y procedimientos para el manejo técnico-administrativo de los servicios educativos para estudiantes de III y IV ciclo de educación especial en colegios técnicos y académicos, expresan que "los docentes de Educación Especial le corresponde orientar tanto a los alumnos como a los padres de familia en el caso en que los estudiantes estén utilizando programas de Educación de Adultos o similares para su formación académica" (MEP, 2005, p. 96).

Determino, por lo tanto, indiscutiblemente valioso, necesario y casi de carácter obligatorio, contar con asesorías reales, dirigidas a miembros de familia, por parte de centros que trabajan con adultos con discapacidad.

En las experiencias desde el Proyecto UNA Oportunidad de Empleo (2011-2012) (Holst y Madrigal, 2014) y en las diversas oportunidades de contar con conversaciones informales con padres de familia, expresaban, constantemente, que desconocen los planes de estudio, las especialidades de profesionales a cargo de sus hijos e hijas, los horarios dedicados a áreas académicas y áreas técnicas y las diversas y más adecuadas opciones educativas, según el compromiso de sus hijos e hijas para concluir la secundaria. 
doi: http://dx.doi.org/10.15359/ree.19-2.12

URL: http://www.una.ac.cr/educare

CORREO: educare@una.cr

En el Curso de adolescencia y necesidades educativas especiales de la Carrera de Educación Especial con énfasis en integración de la Universidad Nacional de Costa Rica, especificamente en los años 2011, 2012 y 2013, las estudiantes asistieron a diferentes centros de atención para adultos con discapacidad (CAIPAD) y servicios de III Ciclo y Ciclo Diversificado Vocacional. En estas visitas las alumnas, por medio de informes semanales, sistematizaron, como una significativa debilidad, que los padres y madres de personas adultas con discapacidad no saben evaluar o determinar, si las labores que hace el personal docente del colegio o centro donde asisten sus hijos es el más adecuado y ético, o bien, si son centros que funcionan como guarderías. Estas circunstancias definitivamente constituyen un reto para quienes atendemos la educación especial, como elementos necesarios de abordar, para lograr brindar a la persona con discapacidad la atención que requiere y que merece un esfuerzo conjunto entre docentes y familiares.

De acuerdo con el Centro Nacional de Recursos para la Educación Inclusiva (CENAREC) (2012) en el documento Orientaciones para promover la transición a la vida adulta de estudiantes de III y IV Ciclo en centros de educación especial promueve:

El centro, la familia, los docentes y sus estudiantes deben mantener relaciones de reciprocidad comunicativa, convirtiéndose en aprehendientes al interactuar con otros, como complejos organizativos ... Se intenta incrementar mejoras cualitativas de comunicación e interacción en cada ambiente de organización de los Centros de Educación Especial (p. 54).

Por otra parte, la Ley 7600 en su "Artículo 20- Derecho de los padres de familia reconoce y afirma que a los padres de familia o encargados de estudiantes con discapacidad, se les garantiza el derecho de participar en la selección, ubicación, organización y evaluación de los servicios educativos" (Asamblea Legislativa de la República de Costa Rica, 1996, p. 13).

Retomo, entonces, que los padres y madres deben informarse, para juzgar con criterio el servicio que reciben sus hijos e hijas; y el personal docente de educación especial debe plantearse el reto de ir más allá y trabajar también con la familia para compartir el mismo norte y visión hacia dónde se quiere llegar con el adulto en condición de discapacidad.

Si los padres y madres conocen los principios que rigen la transición a la vida adulta, el movimiento de vida independiente, el círculo de la sobreprotección, las opciones educativas, los planes de estudio y la legislación actual en temática de discapacidad, probablemente nos encontremos con una realidad muy diferente a la que se vive actualmente. El padre y madre no toma decisiones en función de instintos, suposiciones o mitos equivocados, sino que se convierte en un aliado del educador y trabaja para la independencia de su hijo e hija, en armonía con las instituciones o centros. 
La familia debe proveer apoyos, en lugar de anteponer barreras actitudinales en función del empleo y las opciones laborales con las que pueden contar sus hijos. Debe constituirse en una aliada en la confianza y seguridad que el joven necesita y mostrar apertura ante los nuevos retos que irán apareciendo en el camino. Esto es un largo proceso que se construye con perseverancia y lucha ante la demanda de derechos, y nuevos constructos ideológicos y sociales en función de la visión que tenemos hacia la persona con discapacidad.

A criterio personal, las universidades, como entes formadores de docentes en educación especial, son los mayores responsables en su idónea capacitación, no solo en el buen dominio temático de los planes educativos y de atención para adultos con discapacidad, sino también en la obligatoriedad y necesidad que retoma incluir a la familia en cualquier trabajo con la persona en esta condición.

\section{Conclusiones}

Cuando nos referimos a procesos de inclusión para las personas con discapacidad, estamos retomando su participación plena en todas las áreas de la vida, por lo tanto, cualquier barrera física y actitudinal que se presente arbitrariamente es digna de ser un tema de trabajo por parte de especialistas y profesionales.

Lo anterior lanza un reto a profesionales de educación especial, en el trabajo arduo y perseverante con las familias. Es necesario contar, por lo tanto, con toda una guía curricular y pedagógica, dirigida a la capacitación y empoderamiento de padres y madres de familia. Estos deben ser indiscutiblemente aliados potenciales, en los procesos de inclusión laboral de sus hijos e hijas y ser, al igual o más que muchos de los docentes, los más importantes motivadores en la posible realidad de que consigan un puesto laboral.

De acuerdo con el documento Transición a la vida adulta de jóvenes con discapacidad múltiple y sordoceguera. América Latina (Buceta et al, 2014), uno de los aspectos con que debe trabajarse para lograr una transición satisfactoria es la familia, ya que esta debe promover, orientar y apoyar en los siguientes aspectos:

- Acceso al conocimiento de lo que implica el proceso de Transición a la vida adulta. ...

- Respetar la cultura, necesidades y tiempos de las familias....

- Acceso al conocimiento y participación de Asociaciones que los nuclean y representan. ...

- Acceso a los apoyos necesarios... (p. 6) 
doi: http://dx.doi.org/10.15359/ree.19-2.12

URL: http://www.una.ac.cr/educare

CORREO: educare@una.cr

Las universidades como parte de los procesos formativos han de ampliar, en su malla curricular, el trabajo de personas adultas en condición de discapacidad, indiferentemente de cual sea la carrera a cursar. Como se expuso anteriormente en el modelo social, la discapacidad es un asunto de todos los seres humanos y una buena parte de las barreras que se desarrollan y evidencian en los diferentes contextos sociales pueden ser derribadas por medio de la adecuada información y capacitación, aunque esta pueda ser parcial en algunas carreras.

La Ley 7600 Igualdad de oportunidades para personas con discapacidad en su Artículo 58.- Temáticas sobre discapacidad establece: "Para garantizar el derecho de todos al desarrollo, los centros de educación superior deberan incluir contenidos generales y específicos sobre discapacidad pertinentes a las diferentes áreas de formación, en la currícula de todas las carreras y niveles (Asamblea Legislativa de la República de Costa Rica, 1996, p. 21).

A partir de las experiencias desarrolladas junto con estudiantes de la Carrera de Educación Especial de la Universidad Nacional de Costa Rica, que llevan dentro de su plan de estudios el curso denominado "Adolescencia y necesidades educativas especiales" de la Carrera de Educación Especial de la Universidad Nacional, se logra contar con una muestra de 120 docentes del año 2010 al 2013, que fueron observados trabajando en servicios de atención para adultos, como son el Tercer Ciclo y Ciclo Diversificado Vocacional ubicados en los colegios regulares del país y los centros de atención integral para adultos con discapacidad (CAIPAD).

Los resultados de crónicas y observaciones que presentaron semana a semana son alarmantes, en tanto la gran mayoría de profesionales a cargo de los servicios mencionados anteriormente no parecen contar con el empoderamiento idóneo para el trabajo tan delicado e importante que se debe hacer con la persona adulta con discapacidad. Esto podría deberse, entre otras causas, a la falta de capacitación y formación por parte de los entes de educación superior y también a una posible carencia en la responsabilidad por parte del Ministerio de Educación Pública, en el nombramiento de profesionales no aptos para tales puestos de trabajo.

Insto, en este punto, a reflexionar que si hacemos un recuento en la historia educativa de una persona con discapacidad, desde los servicios que recibe en estimulación temprana, servicios de apoyo en centros de educación regular o de educación especial, el colegio se conviente en el puente de salida a posibles espacios de participación plena, por medio del desarrollo en el mundo laboral.

Es por lo tanto significativo valorar y retomar cómo muchos niños y niñas con discapacidad reciben excelentes apoyos y estos apoyos deben continuar con la misma calidad cuando son participes de programas para la atención en el momento en que pasan a ser de adultos. Un buen dominio en los planes de estudio y programas de atención, junto con los debidos apoyos familiares, pueden, sin duda alguna, generar resultados valiosos y éxitosos en todo sentido. 
La necesidad y urgencia que merece la creación de escuelas o planes de formación para padres de personas con discapacidad en edades escolares y adolescentes podria ir paulatianemente eliminando y previniendo en las nuevas generaciones de padres, las ideas erradas y estigmas nocivos que, por tanto tiempo e históricamente, han venido opacando y violentado su desarrollo pleno.

Al concluir este ensayo, replanteo mi interés en rescatar que cada persona involucrada en los procesos de inclusión laboral de la persona adulta con discapacidad, coordine y trabaje de manera conjunta con la familia, por el beneficio absoluto y pleno de ese ser humano. Es nuestra responsabilidad ir creando juntos una red de aliados, cada vez más amplia y robusta en la visión, el abordaje y el trabajo de la discapacidad, como constructo social, donde el compromiso por derribar barreras sea un asunto de todos y todas, y donde el beneficio y bienestar de las otras personas, me involucre como una ciudadana y ciudadano activo, en una nación que, según la encuesta del 2012 de Happy Planet, es catalogada por segunda vez consecutiva, como el país más feliz del mundo (New Economics Foundation, 2012), pero que en la cotidianidad apela a la indiferencia ante muchos seres humanos y a la vivencia de una felicidad individual de un gran colectivo, que está muy lejana de responder a una Costa Rica accesible y de coherentes acciones de equidad.

\section{Referencias}

Asamblea Legislativa de la República de Costa Rica. (29 de mayo de 1996). Ley 7600 Igualdad de oportunidades para las personas con discapacidad. San José, Costa Rica: Diario Oficial La Gaceta $N^{\circ}$ 112. Recuperado de http://www.munialajuela.go.cr/app/documentos/LEY7600.pdf

Asamblea Legislativa de la República de Costa Rica. (29 de setiembre de 2008). Ley 8661. Aprobación de la Convención sobre los Derechos de las personas con discapacidad. San José, Costa Rica: La Gaceta 187.

Buceta, C., Burgés, H., Ferioli, G., Laynes, M. G., López, R. .. So Soza, Á. M. (2014). Transición a la vida adulta de jóvenes con discapacidad múltiple y sordoceguera. America Latina. Madrid: FOAL Perkins Internacional América Latina. Recuperado de http://www.ciapat.org/biblioteca/pdf/987Transicion a la vida adulta de jovenes con discapacidad multiple y sordoceguera america latina.pdf

Centro Nacional de Recursos para la Inclusión Educativa (CENARC). (2012). Orientación para promover la transición a la vida adulta de estudiantes III y IV ciclo en centros de educación especial. San José: Procesos Litográficos de Centroamérica. 
doi: http://dx.doi.org/10.15359/ree.19-2.12

URL: http://www.una.ac.cr/educare

CORREO: educare@una.cr

Córdoba, L., Mora, A., Bedoya, Á.y Verdugo, M. Á. (2007). Familias de adultos con discapacidad intelectual en Cali, Colombia. Desde el modelo de la calidad de vida. PSYKHE, 16(2), 29-42. Recuperado de http://www.scielo.cl/pdf/psykhe/v16n2/art03.pdf

Gutiérrez, M. L. (2006). Fuertes y capaces: Estrategias metodológicas para el trabajo con niños, niñas y adolescentes con discapacidad. San José, Costa Rica: Ediciones Proniñ@. Recuperado de http:// paniamor.org/ literature 49967/Fuertes y Capaces.

Holst, B.y Madrigal, M. (2014). Proyecto UNA OportunidaddeEmpleo (Informefinal). Heredia:Universidad Nacional de Costa Rica.

Instituto Nacional de estadística y Censos Costa Rica (INEC). (2011). Censo de Población de Costa Rica. Recuperado de http://www.inec.go.cr/Web/Home/GeneradorPagina.aspx

Leach, S. (2002). Empleo con apoyo: Buenas prácticas basadas en principios claros. En M. Á. Verdugo y B. J. de Urríes (Coords.), Hacia la integración plena mediante el empleo (pp. 13-34). Salamanca: KADMOS. Recuperado de http://campus.usal.es/ inico/newsletter/actasempleo.pdf

López, R. (2011). Evolución histórica y conceptual de la discapacidad y el respaldo jurídicopolítico internacional. El paradigma de los derechos humanos y la accesibilidad. Alteridad, Revista de Educación, 6(2), 102-108. Recuperado de http://alteridad.ups.edu.ec/ documents/1999102/3570292/v6n2 Lopez.pdf

Ministerio de Educación Pública (MEP). (2005). Normas y procedimientos para el manejo técnicoadministrativo de los servicios educativos para estudiantes de III y IV ciclo de educación especial en colegios técnicosyacadémicos. San José, Costa Rica: División Desarrollo Curricular, Departamento de Educación Especial, Sección de Desarrollo Vocacional.

Ministerio de Trabajo y Seguridad Social de Costa Rica. (2012). Plan nacional de inserción laboral para la población con discapacidad en Costa Rica. San José, Costa Rica: Autor. Recuperado de http:// www.pnud.or.cr/images/stories/planmaf.pdf

New Economics Foundation. (2012). The Happy Planet Index: 2012 Report [índice del planeta feliz: 2012 informe]. Recuperado de http://www.happyplanetindex.org/assets/happy-planetindex-poster.pdf

Silva, M. (2013) Habilidades blandas, fundamentales para el desarrollo personal. Revista Educar. Recuperado de http://www.cide.cl/documentos/Revista educar Habilidades blandas MJValdebenito.pdf

Vargas, M. (2012). Dimensión político-ideológica de la discapacidad: Un análisis desde el enfoque socio-crítico. Revista Electrónica Educare, 16(2), 171-183. Recuperado de http://www.revistas. una.ac.cr/index.php/EDUCARE/article/view/3938 
doi: http://dx.doi.org/10.15359/ree.19-2.12

URL: http://www.una.ac.cr/educare

Verdugo, M. Á. (2000). Educación y calidad de vida: La autodeterminación de alumnos con necesidades educativas especiales. En IIICongreso "La atención a la diversidad en el sistema educativo"(pp. 1-9). Salamanca: Universidad de Salamanca. Instituto Universitario de Integración en la comunidad. Recuperado de https://campus.usal.es/ inico/actividades/actasuruguay2001/14.pdf

\section{(6) Cómo citar este artículo en APA:}

Madrigal-Lizano, A. M. (Mayo-agosto, 2015). El papel de la familia de la persona adulta con discapacidad en los procesos de inclusión laboral: Un reto para la educación especial en Costa Rica. Revista Electrónica Educare, 19(2), 197-211. doi: http://dx.doi.org/10.15359/ree.19-2.12

Nota: Para citar este artículo en otros sistemas puede consultar el hipervínculo "Como citar el artículo" en la barra derecha de nuestro sitio web: http://www.revistas.una.ac.cr/index.php/EDUCARE/index 